\title{
How does value distract?
}

\author{
When making economic decisions, our choices are often influenced by irrelevant information. One prominent \\ explanation appeals to normalisation in neural circuits. A new paper by Gluth and colleagues suggests that instead, \\ attentional processes may be responsible.
}

\section{Christopher Summerfield and Tsvetomira Dumbalska}

T he human brain is a relativist. Popular sensory illusions reveal how everyday sensations-colours, sounds or tastesare evaluated relative to the context in which they occur. A more striking claim, however, is that more cognitive or economic decisions (such as those about consumer goods or gambles) are similarly shaped by irrelevant context. Influential literature has revealed that decisions among economic goods may reverse in the presence of 'decoy' alternatives that are irrelevant by virtue of being dispreferred or unavailable. For example, the offer of rosé wine may prompt a restaurant customer to switch their choice from white to red, even if rosé itself is never in contention ${ }^{1}$.

New work by Gluth and colleagues ${ }^{2}$ in Nature Human Behaviour calls for a reassessment of a very influential theory ${ }^{3}$ of why decoy effects occur. The theory proposes that neural signals for competing economic alternatives with value $v A, v B$ and $v C$ are divisively normalised, so that the neural response to $A$ is proportional to $\frac{v A}{v A+v B+v C}$. This mirrors a principle ubiquitously observed in neural circuits ${ }^{4}$. The normalisation model predicts that for an agent with preference ordering $v A>$ $v B>v C$, the probability $p(A)$ of choosing option $A$ over $B$ depends on influence of the distracter item $C$-a violation of rational choice theory ${ }^{5}$. In 2013, important behavioural support for this model was provided by the report of a new decoy effect that matches this prediction: when humans or monkeys made decisions between three primary reinforcers, $p(A)$ shrinks as $v C$ grows ${ }^{3}$. This 'distracter effect' is predicted by the normalisation model because the denominator increases with $v C$, leading to stronger normalisation, reducing the difference in neural signals elicited by $A$ and $B$ and rendering them more liable to confusion. The behavioural finding has been cited more than 100 times, is supported by additional data from other domains (for example, facial attractiveness ${ }^{6}$ ) and been used to constrain related theories ${ }^{7}$. The original paper has been influential because it draws a compelling link between violations of rationality and canonical computational principles in neural circuits.

However, in new work, Gluth and colleagues report a convincing failure to replicate the human distracter effect ${ }^{2}$. In a cohort of over 100 participants, they report no influence of the value of item $C$ on human choice accuracy between $A$ and $B$. The study is distinguished by its unusually wellplanned and rigorous methods. The authors preregistered their study, consulted with the original authors (who were extremely helpful and responsive) to ensure that their code was identical, carefully selected their sample size to have sufficient power to demonstrate a potential null finding, used Bayesian model selection to establish the probability of a lack of effect, replicated their own failure of replication in a comparable data set from another lab ${ }^{8}$ and even identified a potential weakness in one analysis conducted in the original study that may have inflated the likelihood of their finding a positive effect. Anyone wishing to conduct a replication study should consult this paper: it provides an invaluable roadmap for how to proceed and is a textbook example of best practice in the cognitive sciences.

So where does this leave the divisive normalisation model for economic decisions? In their data, Gluth et al. failed to see an influence of $v C$ on choices between $A$ and $B$, but they did-intriguingly_-find that response times (RTs) slowed as $v C$ increased. In other words, while the original paper reports a distracter effect in choice but not RT, the new paper reports the effect in RT but not choice. By measuring eye movements, Gluth et al. were also able to establish that while both high- and low-valued distracters captured attention early in the trial to similar degrees, attention to low-valued distractors dwindled faster as the trial progressed. Their data were captured by a variant of a previously proposed sequential sampling model $^{8}$, in which deliberation is biased towards the item that is currently fixated, that was modified to allow the gaze is itself to be more readily allocated to valuable items. This allows the model to generate a 'cascade' effect whereby participants both look at what they want and want what they look at ${ }^{9}$. Thus, one possibility is that the choice biases reported in the original paper were at least in part due to a capture of attention by more valuable distracters for longer periods of each trial. Of note, however, this theory would not account for the monkey data (not challenged in the new paper), because the animals responded an order of magnitude faster than the humans but were even more prone to the distracter effect.

The idea that violations of rationality may be secondary to the way that participants allocate attention, rather than to neural activity dynamics, is one that has gained some traction recently ${ }^{10}$. However, divisive normalisation is a well-established principle with a long pedigree in neural circuits, and it seems plausible that it plays a role in evaluating both sensory and cognitive variables relative to the context in which they occur. The question remains open, but these new data from Gluth and colleagues offer a valuable new piece of the puzzle.

\section{Christopher Summerfield (D)* and Tsvetomira Dumbalska \\ Department of Experimental Psychology, University of Oxford, Oxford, UK. \\ *e-mail: christopher.summerfield@psy.ox.ac.uk}

Published online: 3 February 2020 https://doi.org/10.1038/s41562-020-0824-y

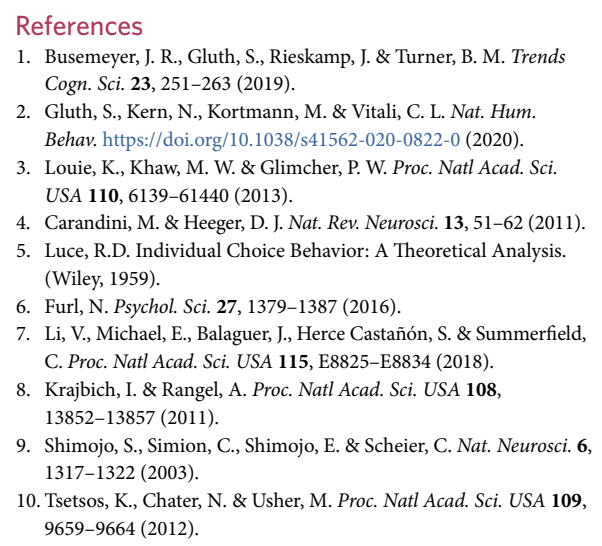

9. Shimojo, S., Simion, C., Shimojo, E. \& Scheier, C. Nat. Neurosci. 6, $1317-1322$ (2003).

10. Tsetsos, K., Chater, N. \& Usher, M. Proc. Natl Acad. Sci. USA 109, 9659-9664 (2012). 